\title{
Distal Molar Surgery
}

\author{
Khushboo Jeevan Durge ${ }^{1}$, Pavan Bajaj ${ }^{2}$, Diksha Agrawal ${ }^{3}$ \\ 1, 2, 3 Department of Periodontics, Sharad Pawar Dental College \& Hospital, Datta Meghe Institute of Medical Sciences \\ (Deemed to Be University), Sawangi (Meghe), Wardha, Maharashtra, India.
}

\section{INTRODUCTION}

Periodontitis is a chronic inflammation involving tooth investing structures. ${ }^{1}$ It causes irreversible bone and attachment loss. Very often third molar impaction leads to various complications adversely influencing the entire periodontal status. ${ }^{2}$ Therefore disimpaction surgery is one of the most commonly performed procedures. Extraction of an impacted molar tends to initiate localized periodontal pockets distally to an adjacent molars. ${ }^{3}$ This favours colonization of the subgingival microbiota, due to the difficulties associated with hygiene, and leads to the appearance of progressive bone loss into an well-defined angular defect. ${ }^{4}$ The primary goal of periodontal therapy is aimed at prevention, slowing or arresting disease progression. ${ }^{5}$ Gingivectomy is the first choice of treatment in distal pocket due bulbous fibrous tissue. It can only be the choice of treatment when adequate amount of keratinized tissue is present at the site. Nevertheless, many times inadequate keratinized tissue or an angular bony defect has been diagnosed. Then bulbous tissue is preferably reduced in bulk and not be removed completely by the distal molar surgery. ${ }^{6}$ The technique aids in pocket management. Apart, it also facilitates access to the osseous defect. It also secures the sufficient amounts of gingival tissue to achieve coverage.

Oral cavity is one of the most complex systems of a human body. It comprises of numerous undersell areas which serve as bacterial plaque retention zones. If not controlled by proper oral hygiene practices, may lead to various periodontal diseases. One such strenuous clinical situation reported in many cases is the management of deep periodontal pockets on the distal aspect of molar as a sequel of disimpaction procedure. The scenario is even more complicated due to the bulbous tissues over the tuberosity or prominent retro-molar pad. Therefore, in this case report distal molar surgery using triangular incision was performed followed by uneventful healing of surgical site. The outcome of this case suggests that, in an attempt to manage and prevent the further loss of periodontal structures around molar adjacent to third molar, distal molar surgery has proven to be a simple and promising procedure.

This procedure is also termed as distal wedge procedure. It was originally given by Robinson in $1966 .{ }^{6}$ It is indicated on the distal aspect of terminal molars or on other proximal tooth surfaces encircling edentulous spaces

a. For treating periodontitis,

b. Enabling an effective oral hygiene practice,

c. An ease in restorative aspects.

The procedure can be performed as an independent plan of action or in adjunctive with various periodontal therapies like flap access for crown lengthening and apically positioned flap. Numerous osseous and regenerative surgery can also be accompanied with the same. ${ }^{7}$

\section{Contraindications}

a. Close proximity to an ascending ramus,

b. Existing prominent external oblique ridge. ${ }^{8}$

\author{
Corresponding Author: \\ Dr. Khushboo Durge. \\ Department of Periodontics, Sharad Pawar \\ Dental College \& Hospital, Datta Meghe \\ Institute of Medical Sciences (Deemed to Be \\ University), Sawangi (Meghe), Wardha, \\ Maharashtra, India. \\ E-mail: khushboodurge5@gmail.com
}

DOI: $10.14260 / j e m d s / 2020 / 732$

How to Cite This Article:

Durge KJ, Bajaj P, Agrawal D, et al. Distal molar surgery. J Evolution Med Dent Sci 2020;9(44):3335-3338,

$10.14260 /$ jemds $/ 2020 / 732$ DOI:

Submission 16-07-2020,

Peer Review 25-09-2020,

Acceptance 01-10-2020,

Published 02-11-2020.

Copyright (c) 2020 Khushboo Jeevan Durge et al. This is an open access article distributed under Creative Commons Attribution License [Attribution 4.0 International (CC BY 4.0)] 
However certain modifications are also reported in the literature which can be performed based on the anatomical variations and clinical situations varying per se patient. (Figure 3 and Figure 4).

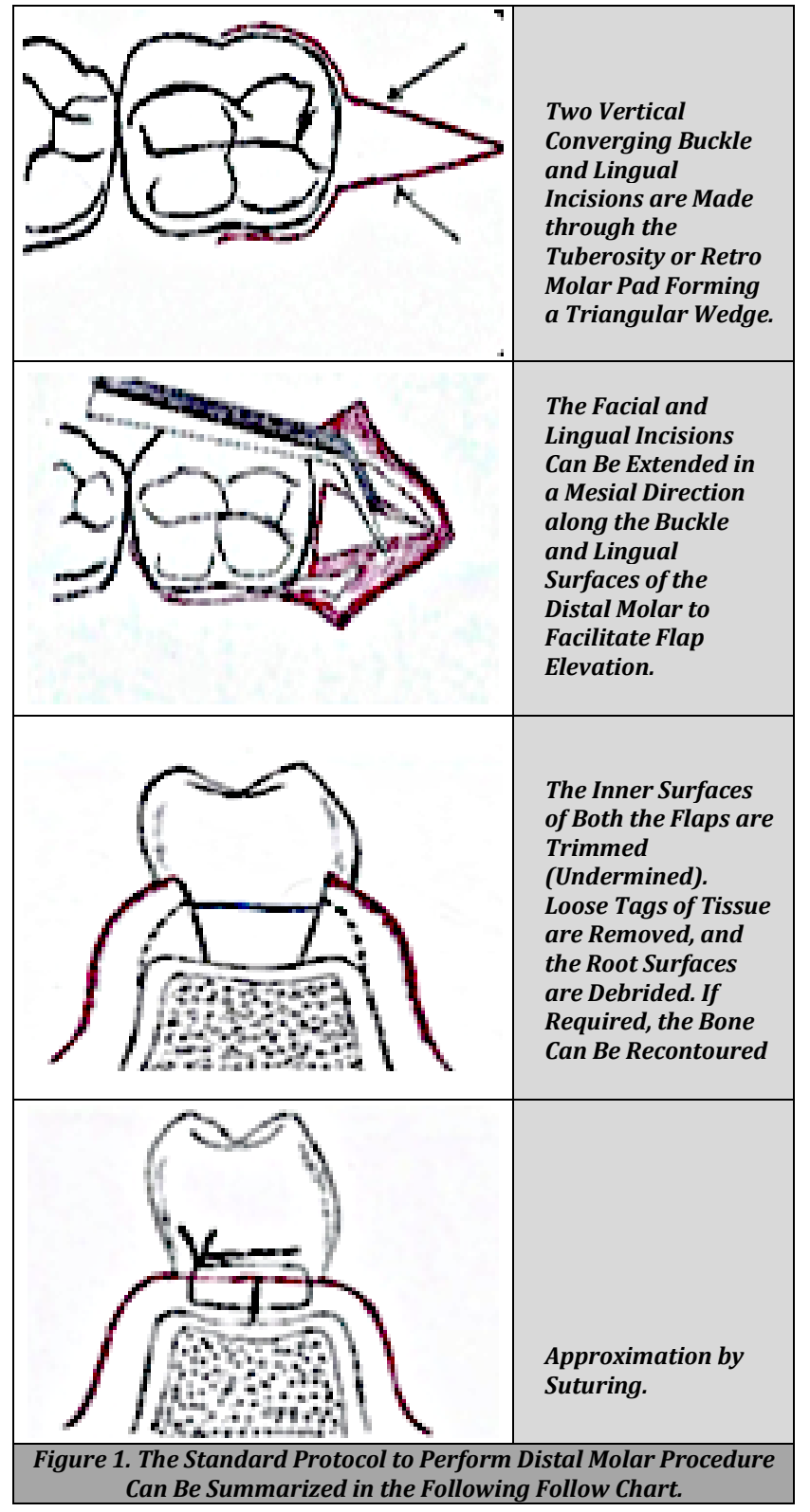

Various Modifications of Incisions in Distal Molar Surgery

There are three typical incision designs routinely followed are rectangle, triangular, and linear (Line).

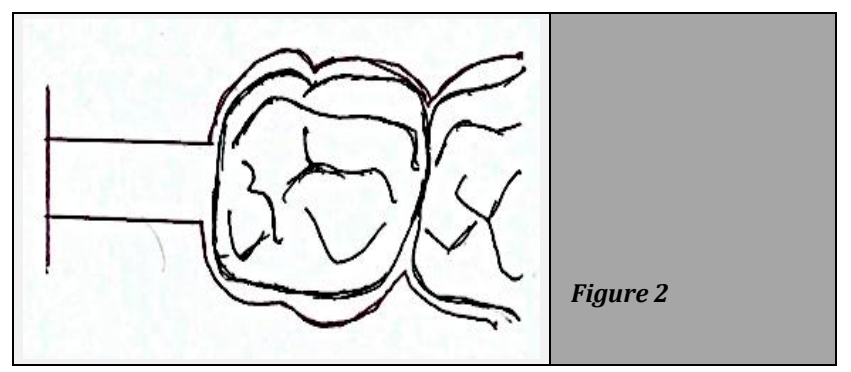

When a combination of a deep periodontal pocket with an angular bone defect at the distally to maxillary molar the rectangular incision variation can used. (Figure 2) Two parallel reverse bevel incisions, one buccal and one palatal, can be made from the distal surface of the molar to the posterior part of the tuberosity, where they are connected with a buccolingual incision.

The buccal and palatal incisions are extended in a mesial direction along the buccal and palatal surfaces of the molar to facilitate flap elevation.
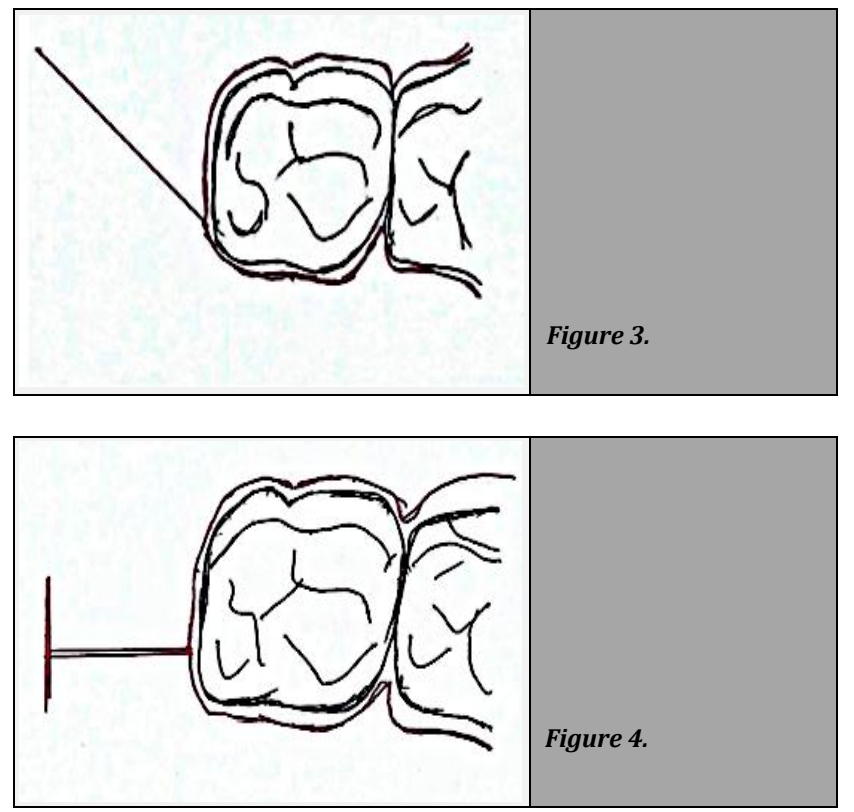

These incisions can be practice to ensure optimal flap adaptation at the furcation site. The amount of attached keratinized tissue present as well as the accessibility to the retro-molar area has to be considered when placing the incision.

\section{PRESENTATION OF CASE}

A 35-year-old female patient reported to the Department of Periodontics. Patient complaints chiefly a mild to moderate pain in her lower left back region of jaw since 2 to 3 months. The patient also revealed food lodgement with the same. A detailed case history was taken. Past dental history showed that she went through dis-impaction procedure with lower left third molar before 10 months. On periodontal examination a deep distal pocket measuring $6 \mathrm{~mm}$ with lower left second molar was seen. Initial periodontal therapy including scaling and root planing was carried out. On 6-week follow-up the inflammatory component was subsided. On re-examination the pocket depth was still $6 \mathrm{~mm}$ with mild discomfort in the patient (Figure 5). The residual pocket depth was taken into accordance. Therefore, in ordered to manage the distal pocket and probable underlying osseous defect it was decided to proceed with distal wedge procedure.

\section{DISCUSSION OF MANAGEMENT}

\section{Surgical Phase}

Prior to surgical procedure a written signed informed consent was taken from them. An area was anesthetized with a solution 
of $2 \%$ lignocaine ${ }^{9}$ with 1:200,000 adrenaline under all aseptic precautions. Two vertical incisions were made on either sides buccally and lingually meeting at one point in the retro-molar region forming a rough triangular wedge. (Figure 6). The crevicular incision was then extended till second molar mesially. Then the full thickness muco-periosteal flap ${ }^{10}$ was elevated. The underlying bone and root surfaces were debrided thoroughly to remove diseased granulation tissue if any and the bulbous fibrous tissue was trimmed of reduce the pocket depth (Figure 7). The facial and lingual surfaces was then undermined. The flaps were then approximated by simple interrupted sutures with 3 - 0 silk material (Figure 8).
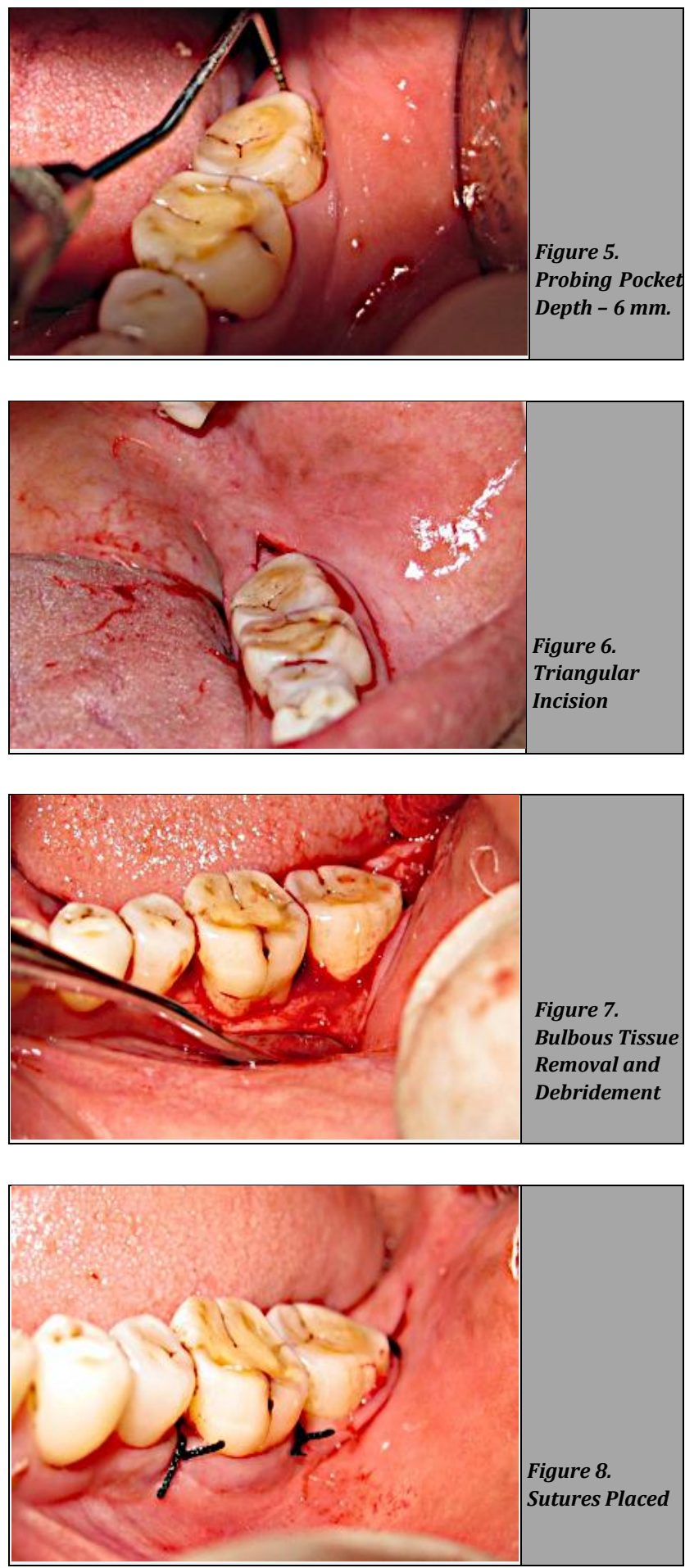

\section{Post-Operative Care}

All the post-operative instructions were given. Patient was advised antibiotics (amoxicillin, $500 \mathrm{mg}$ TID) and analgesics after surgery for 5 days. Patient was encouraged to practice intermittent cold fermentation for next 24 hours. ${ }^{11}$ Suture was removed within 7 to 10 days. Patient was instructed not to brush in the treated area and to rinse 3 times per day for 1 minute with chlorhexidine digluconate $0.12 \%$ until suture removal. Patient was advised to maintain a soft diet for at least 2 weeks.

\section{DISCUSSION}

During the extraction, movement of the tooth can be recognized in an unfavourable direction. So, it is advised to prefer an open surgical procedure rather than using elevators. ${ }^{12}$ This over-instrumentation may result into future initiation of periodontal osseous defects. A removal of impacted molar often results in increased risk for caries and periodontal intrabony defects adjacent to the distal surface of the second molar. ${ }^{13,14}$

A retrospective study have showed after 2 years, $43 \%$ of the cases as a consequence of surgical removal of the impacted molar had periodontal pocket $\geq 7 \mathrm{~mm}$ distally to adjacent molar. ${ }^{15}$ In the present case report, although the patient did not have any periodontal disease earlier, but on periodontal examination showed deep distal pocket around $5 \mathrm{~mm}$ to lower left second molar, 10 months following third molar extraction.

Studies have revealed, periodontitis at the distal side of terminal molars along with anatomic factors may increase periodontal probing depth. ${ }^{6,17,18,19}$ Therefore, considering the findings through previous studies with a view to maintain the periodontal health status of patient distal molar surgery was performed.

The results of our case report showed reduced pocket depth to $3 \mathrm{~mm}$. Also, preservation of existing keratinized (attached) tissue or either increasing width of keratinized (attached) tissue is of paramount importance in periodontal therapy. The gingival tissue enclosing the distal aspect may attribute to increase the zone of attached gingiva. This is due to granulation tissue contributions from wounded gingival connective tissue. ${ }^{20}$ Advocating previous studies our case report also showed improved keratinized (attached) tissue postoperatively.

\section{CONCLUSIONS}

Dental plaque is one of the commonest aetiologic agents which has been reported to worsen various systemic conditions. Surpassingly, in an attempt to manage deep periodontal pocket, the major reservoir of bacterial plaque can be efficiently coped with distal molar surgery. The present case report shows that distal molar surgery can still be a simple periodontal surgery for the management of periodontal pocket distal to molar. It may also contribute to arrest the predicted bone loss.

Financial or other competing interests: None.

Disclosure forms provided by the authors are available with the full text of this article at jemds.com. 


\section{REFERENCES}

[1] Agrawal DR, Jaiswal P, Masurkar D. Gingival fibroma: report of two cases with different treatment modalities. Journal of Medical Science 2020;24(104):2604-09.

[2] Patil C, Jadhav A, Rajanikanth K, et al. Piezosurgery vs. bur in impacted mandibular third molar surgery: evaluation of postoperative sequelae. Journal of Oral Biology and Craniofacial Research 2019;9(3):259-62.

[3] Khzam N, Fell A, Fisher A, et al. A new multi-ingredient recipe for the treatment of localized advanced periodontal disease following the surgical removal of impacted wisdom teeth. Case Reports in Dentistry 2016;2016:3847615.

[4] Leung WK, Corbet EF, Kan KW, et al. A regimen of systematic periodontal care after removal of impacted mandibular third molars manages periodontal pockets associated with the mandibular second molars. J Clin Periodontol 2005;32(7):725-31.

[5] Wang HL, Greenwell H. Surgical periodontal therapy. Periodontology 2000 2001;25(1):89-99.

[6] Robinson RE. The distal wedge operation. Periodontics 1966;4(5):256-264.

[7] Johnson TM, Herold RW, Akers JA, et al. Decisions before incisions: technique selection for the distal wedge procedure. Clinical Advances in Periodontics 2020;10(2):94-102.

[8] Lindhe J, Lang NP, Karring T. Clinical periodontology and implant dentistry. $4^{\text {th }}$ edn. Copenhagen: Blackwell Munksgaard 2003.

[9] Reddy $\mathrm{KV}$, Jadhav $\mathrm{A}$, Bhola $\mathrm{N}$, et al. Is $0.75 \%$ ropivacaine more efficacious than $2 \%$ lignocaine with $1: 80,000$ epinephrine for IANB in surgical extraction of impacted lower third molar? Oral and Maxillofacial Surgery (Heidelberg) 2019;23(2):225-31.

[10] Borle R, Nimonkar P, Hingnikar P, et al. Efficacy and safety of different interpositional flaps and grafts for closing fibrotomy wound in patients of oral submucosal fibrosis: a systematic review with meta-analysis. Medical Science 2019;23(100):1031-48.

[11] Greenstein G. Therapeutic efficacy of cold therapy after intraoral surgical procedures: a literature review. Journal of Periodontology 2007;78(5):790-800.

[12] Jain A. Accidental displacement of mandibular first molar root into buccal space: a unique case. Journal of Stomatology Oral and Maxillofacial Surgery 2018;119(5):429-31.

[13] Faria AI, Gallas-Torreira M, Lopez-Raton M, et al. Radiological infrabony defects after impacted mandibular third molar extractions in young adults. Journal of Oral and Maxillofacial Surgery 2013;71(12):2020-8.

[14] Irja V. Impacted third molars increase the risk for caries and periodontal pathology in neighboring second molars. Journal of Evidence-Based Dental Practice 2014;14(2):89-90.

[15] Kugelberg CF, Ahlstrom U, Ericson S, et al. Periodontal healing after impacted lower third molar surgery. A retrospective study. International Journal of Oral Surgery 1985;14(1):29-40.

[16] Braden BE. Deep distal pockets adjacent to terminal teeth. Dent Clin North Am 1969;13(1):161-8.

[17] Müller HP, Schaller N, Eger $T$, et al. Thickness of masticatory mucosa. J Clin Periodontol 2000;27(6):431-6.

[18] Horning GM, Cohen ME, Neils TA. Buccal alveolar exostoses: prevalence, characteristics, and evidence for buttressing bone formation. J Periodontol 2000;71(6):1032-42.

[19] Kramer GM, Schwarz MS. A technique to obtain primary intention healing in pocket elimination adjacent to an edentulous area. Periodontics 1964;2:252-7.

[20] Karring T, Cumming BR, Oliver RC, et al. The origin of granulation tissue and its impact on postoperative results of mucogingival surgery. J Periodontol 1975;46(10):57785. 